\title{
Typ-2-Diabetes \\ Insulineinstieg ohne erhöhte Gefahr für Hypoglykämien oder Gewichtszunahme
}

- Das Basalinsulin Insulindetemir ist nun als Add-on bei Typ-2-Diabetikern, die mit dem GLP-1-Analogon Liraglutid und Metformin behandelt werden, zugelassen. Durch Zugabe des Insulinanalogons wird das $\mathrm{HbA}_{1 \mathrm{c}}$ weiter gesenkt, ohne das Risiko für schweren Unterzucker und Gewichtszunahme zu steigern.

In der zulassungsrelevanten Studie erhielten 821 mit Metformin und z.T. mit Sulfonylharnstoff vorbehandelte Typ-2-Diabetiker zunächst in einer zwölfwöchigen Vorlaufphase Metformin und einmal täglich Liraglutid (Victoza ${ }^{\circledast}$ ), so der niedergelassene Diabetologe Dr. Jörg Steindorf aus Schkeuditz. Patienten, deren $\mathrm{HbA}_{1 \mathrm{c}}$ so unter $7 \%$ fiel, wurden nach Randomisierung entweder über 26 Wochen derartig weiterbehandelt $(\mathrm{n}=161)$ oder sie erhielten zusätzlich einmal täglich Detemir (Levemir ${ }^{\circledast}, \mathrm{n}=162$ ).

Die Eskalation mit dem Basalinsulin über diesen Zeitraum führte zu einer zusätzlichen $\mathrm{HbA}_{1 \mathrm{c}}$-Senkung um 0,5\%-Punkte ohne schwere Hypoglykämien. Zusätzlich bemerkenswert war die anhaltende und mit Detemir sogar um $0,5 \mathrm{~kg}$ noch verstärkte Gewichtsabnahme von insgesamt $4 \mathrm{~kg}$ über ein Jahr, so Steindorf. Patienten, die wegen der guten $\mathrm{HbA}_{1 c^{-}}$ Werte in der Vorlaufphase nicht randomisiert werden mussten $(\mathrm{n}=498)$, erreichten nach 26 Monaten den kombinierten Endpunkt ( $\mathrm{HbA}_{1 c}$-Ziel erreicht, keine Gewichtszunahme, keine schwere Hypoglykämie), etwa doppelt so oft wie die der randomisierten Gruppen. Für Steindorf ist dies ein Hinweis darauf, dass die frühzeitige Kombitherapie mit einem GLP-1Analogon die BZ-Einstellung deutlich verbessern kann. Indiziert ist diese Therapie v.a. bei übergewichtigen Patienten und unzureichender Stoffwechsellage bei Metformin-Monotherapie oder einer oralen Kombitherapie, so PD Dr. Rainer Lundershausen von der Median-Klinik Bad Berka. Zudem profitieren Patienten, für die Hypoglykämien ein besonderes Risiko sind, z.B. beruflich oder wegen Herzerkrankung. Wird das individuelle Therapieziel trotz des GLP-1-Analogons nicht erreicht, ist die Kombination mit Detemir sinnvoll, so Lundershausen. Das Basalinsulin sollte, unter Beibehaltung der Vortherapie mit Liraglutid und Metformin, zunächst mit zehn Einheiten niedrig dosiert abends eingesetzt und die Dosis anhand der Nüchtern-Blutzuckerwerte alle drei bis fünf Tage entsprechend angepasst werden. hae

Quelle: Presseworkshop am 16.2.2012 in Frankfurt a. M., Veranstalter: Novo Nordisk

\section{Netzhauterkrankungen Bedarfsgerechte Therapie je nach Visusverlauf}

- Bei altersbedingter Makuladegeneration (AMD), dem Diabetischen Makulaödem (DMÖ) und dem Makulaödem aufgrund eines Retinalen Venenverschlusses (RVV) hat sich ein patientenangepasstes, flexibles Behandlungsschema durchgesetzt.

Durch die Zulassung des VEGF-Inhibitors Ranibizumab (Lucentis ${ }^{\circledast}$ ) für die drei Indikationen exsudative AMD, DMÖ und RVV hat sich das Management von Netzhauterkrankungen deutlich verbessert. In den letzten Jahren hat sich gezeigt, dass sich das Ausmaß der zugrundeliegenden Überexpression des Wachstumsfaktors VEGF zwischen den drei Netzhauterkrankungen und auch interindividuell unterscheidet.

Dem individuellen Krankheitsverlauf werde man heute durch ein bedarfsgerechtes, auf den individuellen Patienten zugeschnittenes Behandlungsschema (Pro-re-nata, PRN) gerecht, berichtete Prof. Dr. Gabriele Lang von der Klinik für Augenheilkunde am Uniklinikum Ulm. Das PRN-Schema habe das Ziel, den bestmöglichen Behandlungseffekt bei geringster Belastung des Patienten zu erreichen. Laut Lang er- reichen die meisten Patienten innerhalb von drei Monaten ihre maximalen Visusergebnisse. Meistens werde dieser Therapieerfolg mit weiteren monatlichen Injektionen stabilisiert, aber nicht verbessert. Dem PRN-Schema zufolge wird Ranibizumab zunächst einmal monatlich injiziert, und zwar solange, bis der maximale Visus erreicht ist. Das ist gegeben, wenn an drei aufeinanderfolgenden monatlichen Kontrollen kein weiterer Anstieg der Sehschärfe festgestellt wird. Bei stabilem Visus wird die Therapie abgebrochen, der Patient aber weiterhin engmaschig kontrolliert. Eine Wiederbehandlung ist nötig, wenn sich der Visus um fünf oder mehr Buchstaben verschlechtert. Entscheidendes Kriterium für die Therapieunterbrechung ist also die Stabilität und für die Wiederbehandlung die Instabilität der Sehschärfe. Das flexible Therapieschema erspare stabilen Patienten unnötige Injektionen und sei somit auch kosteneffektiv, so Lang.

aam

Quelle: Presseworkshop am 1.2.2012 in Bad Saulgrub, Veranstalter: Novartis 